\title{
Pathological Role of Fungal Biofilms in Fungal Rhinosinusitis: A Case-Control Study
}

\author{
Waleed F. Ezzat1, Anas Askoura1, Hesham Falah², Ahmed Negm² \\ ${ }^{1}$ Department of Otolaryngology/Head and Neck Surgery, Faculty of Medicine, Ain Shams University, Cairo, Egypt \\ ${ }^{2}$ Department of Otolaryngology/Head and Neck Surgery, Misr University for Science and Technology, Cairo, Egypt \\ Email: wfezzat@ayhoo.com, anas_askoura@med.asu.edu.eg, heshamyehia1991@gmail.com,negm_oto@yahoo.com
}

How to cite this paper: Ezzat, W.F., Askoura, A., Falah, H. and Negm, A. (2021) Pathological Role of Fungal Biofilms in Fungal Rhinosinusitis: A Case-Control Study. International Journal of Otolaryngology and Head \& Neck Surgery, 10, 75-84.

https://doi.org/10.4236/ijohns.2021.102008

Received: October 19, 2020

Accepted: March 1, 2021

Published: March 4, 2021

Copyright $\odot 2021$ by author(s) and Scientific Research Publishing Inc. This work is licensed under the Creative Commons Attribution International License (CC BY 4.0).

http://creativecommons.org/licenses/by/4.0/

(c) (i) Open Access

\begin{abstract}
Objective: This case-control study aimed to detect the presence of fungal biofilms in cases of fungal sinusitis, trying to find its role in recurrence of fungal sinus infection and resistance to medical treatment. Patients and Methods: This study was conducted at Ain-Shams University from June-2017 to June-2018 upon 20 patients as the fungal cases are not much in Egypt. All patients were subjected to functional endoscopic sinus surgery and taking specimens. Examination of specimens by scanning electron microscope was made. Results: The prevalence of fungal biofilms was 7 (70\%) in cases and 0 $(0 \%)$ in controls ( $\mathrm{p}$-value $=0.001$ ). There is higher risk of fungal biofilms in cases of fungal sinusitis, the odds of biofilms is higher in the study group 2.33 than in the control group 0 . The lower is the invasiveness, the higher is the fungal biofilms ( $\mathrm{OR}=0.94,95 \% \mathrm{CI} 0.46-4.05)$. Also, the analysis showed that the lower is the recurrence the lower is the fungal biofilms $(\mathrm{OR}=2.25$, 95\% CI 0.15 - 7.93). Conclusion: This study showed the presence of fungal biofilms in different cases of fungal sinusitis whether primary or recurrent, also whether invasive or non-invasive. Studies with large sample size are recommended to get a strong evidence.
\end{abstract}

\section{Keywords}

Fungal Biofilms, Rhinosinusitis, Recurrence, Invasiveness

\section{Introduction}

Among the many interacted factors behind the etiology of chronic rhinosinusitis (CRS); either systemic, local host, environmental factors, the presence of fungal biofilms has emerged during the last two decades as one of the important environmental etiological factors [1]. 
Biofilm includes multiple microorganisms, bacterial and/or fungal, in a one community adhered by an extracellular polymeric substance. Thus, it got several advantages like synergy, metabolic cooperation and resistance, which contribute to its survivability [2]. Else, being a physical barrier between the fungal cells and the antifungal therapy, biofilms lead to enduring fungal colonization in the sinuses even with maximal therapy. Therefore, they permit the chronic persistence of the microorganisms in the nose and sinuses. Hence, treatment became more difficult because of the impaired efficacy of antifungal treatments [3].

A conclusive evidence that biofilms are present on the mucosa of patients with CRS has been presented by Foreman et al. (2009). However, at first, less was known about the constituting species of these biofilms. The most frequently detected organism was Staphylococcus aureus. In addition, about $20 \%$ of CRS patients had characteristic fungal biofilms [4]. Moreover, a vast range of fungal species have been detected in patients with CRS with Aspergillus fumigatus as a frequent pathogen, and Candida species are rarely seen [5] [6] [7].

Furthermore, in the process of development and survival of biofilms, there is an apparent synergy between bacteria and fungi as evidenced in a study upon animal model. The inoculation of Staphylococcus aureus and Aspergillus fumigatus together into the sinuses of the sheep caused formation of biofilms in $80 \%$ of them versus $10 \%$ with the sole inoculation of Aspergillus fumigates. Staphylococcus aureus seems to produce damage to the sinus epithelium that promotes the formation of fungal biofilm by Aspergillus fumigatus [8].

However, fungal biofilms are poorly understood and represent expanding area of research, both within microbiology and otorhinolaryngology. The more the understanding of the role of biofilms in the etiopathogenesis of CRS, the more is our understanding and searching for better management of those cases [4].

Thus, this study aimed to detect the presence of fungal biofilm in cases of fungal sinusitis through scanning by electron microscopy and trial of detection its role in recurrence of fungal infection in different cases of fungal sinusitis and resistance to medical treatment.

\section{Patients and Methods}

This matched case-control study was conducted upon patients with fungal sinusitis aiming to detect the presence of fungal biofilms, trying to find its role in recurrence of fungal sinus infection and resistance to medical treatment. Written informed consent was obtained from all participants with Institutional Review Board Approval. The study was conducted at Ain Shams University tertiary hospital during the period from June 2017 to June 2018.

The study population consisted of 20 patients, 10 cases and 10 controls matched for age and gender.

Case definition: Cases with fungal sinusitis constituted the study group. Invasive or non-invasive fungal sinusitis, either primary or who underwent previous adequate surgery with recurrent fungal infection will be included.

Control group: 10 patients (five patients with non-fungal rhinosinusitis-nasal 
polyps-undergoing endoscopic sinus surgery) and five patients without any symptoms or signs suggestive of rhinosinusitis who were undergoing other nasal surgery like septoplasty or turbinectomy. They are sinus free as proved by CT paranasal sinuses (PNS).

\section{Inclusion Criteria:}

1) Patients with invasive and non invasive fungal sinusitis (primary).

2) Patients who underwent previous adequate surgery with recurrent fungal infection will be included.

3) CT PNS for patients in control group no.2 must be sinus free.

\section{Exclusion Criteria (for all groups):}

1) Ciliary motility disorders.

2) Wegner's granulomatosis and other granulomatous diseases.

3) Sino-nasal malignancy.

In both cases and control group, those with ciliary motility disorders, Wegner's granulomatosis or other granulomatous diseases and sino-nasal malignancy were excluded.

All the 20 patients (cases and control) were subjected to detailed history, complete ENT examination, endoscopic evaluation of the nose, CT scan (and/or) MRI paranasal sinus (PNS) to evaluate the affected sinuses, functional endoscopic sinus surgery under general anesthesia \pm medical treatment and taking specimens. Histopathological examination using special stain periodic acid Schiff (PAS) to detect the fungal hyphae or by fungal culture. Examination of specimens by SEM (Scanning Electron Microscope). Post-operative follow up for 1 year (at 6 months and at 1 year).

Specimens are collected using a sterile technique and were transported in a sterile container within 2 hours. Depending on the specimen and suspected pathogen, inoculation media may vary. Specimens for routine fungal culture were first inoculated in nonselective media such as Sabouraud dextrose brain heart infusion (BHI) agar or plain BHI agar. A blood-containing media such as BHI blood agar improves the sensitivity or recovery of dimorphic fungi. Selective media such as inhibitory mold agar were used for contaminated specimens. Antibiotics were used to make the media selective. Nonselective media were inoculated with selective media, as some organisms may be inhibited by the antibiotics.

Inoculated media were incubated at $25^{\circ} \mathrm{C}-30^{\circ} \mathrm{C}$ in room air for up to 4 weeks. Systemic dimorphic fungi were incubated at $35^{\circ} \mathrm{C}-37^{\circ} \mathrm{C}$. Fastidious organisms were incubated up to 8 weeks. Candida species usually grow well in aerobic bacterial culture media; growth was finalized after a week of incubation, as turnaround time for yeast is about 7 days.

All operations were done under general anesthesia in the Otorhinolaryngology Head and Neck Department, Ain Shams University Hospitals. All operations were done endoscopically with an endoscopic camera and monitor display.

Specimens were collected from the nasal mucosa of the most affected sinus and transported after some maneuvers to the electron microscopy unit. All sam- 
ples were of an appropriate size to fit in the specimen chamber and were generally mounted rigidly on a specimen holder called a specimen stub.

Specimens from patients were rinsed in $2.5 \%$ glutaraldehyde fixative solution buffered with $0.1 \%$ phosphate buffer for 3 hours. Specimens were then post fixed in osmium tetroxide for 15 minutes. For SEM (Scanning Electron Microscopy), specimen was normally required to be completely dry, since the specimen chamber was at high vacuum. Living cells, tissues and soft-bodied organisms usually require chemical fixation to preserve and stabilize their structure.

Tissues were dehydrated through ascending grades of ethanol (from 50\% $90 \%$ concentration) using automatic tissue processor (Leica EM TP) and then were dried at critical point dryer using liquid carbon dioxide with (Tousimis Audosamdri-815) Air-drying cause collapse and shrinkage, this is commonly achieved by replacement of water in the cells with organic solvents such as ethanol or acetone, and replacement of these solvents in turn with a transitional fluid such as liquid carbon dioxide by critical point drying. The carbon dioxide was finally removed while in a supercritical state, so that no gas-liquid interface was present within the sample during drying.

Tissues were further mounted on brass studs with aluminum conducting tape and coated by gold sputter coater (SPI-Module). For conventional imaging in the SEM, specimens must be electrically conductive, at least at the surface, and electrically grounded to prevent the accumulation of electrostatic charge at the surface. Metal objects require little special preparation for SEM except for cleaning and mounting on a specimen stub. Nonconductive specimens tend to charge when scanned by the electron beam, and especially in secondary electron imaging mode, this causes scanning faults and other image artifacts. They are therefore usually coated with an ultrathin coating of electrically conducting material, deposited on the sample either by low-vacuum sputter coating or by high-vacuum evaporation. Conductive materials in current use for specimen coating include gold, gold/palladium alloy, platinum, osmium, iridium, tungsten, chromium, and graphite.

Specimens were examined by scanning electron microscopy (JEOl-JSM-5500 IV) by using high vacuum mode at the Regional Center of Mycology and Biotechnology, Cairo, Egypt. Normally, nasal mucosa appeared under SEM as pseudostratified ciliated columnar cells with goblet cells. In cases of CRS, multiple levels of affection were present (distorted or absent cilia, absent microvilli, absent goblet cells, distorted cells etc.) and this picture may be associated with biofilm presence (positive specimens) or not (negative specimens). Biofilm was present as a layer of exopolysaccharide (EPS) over the mucosa and after magnification, we can see the ultrastructure of biofilm.

\section{Statistical Analysis}

Recorded data were analyzed using the statistical package for social sciences, version 20.0 (SPSS Inc., Chicago, Illinois, USA). Quantitative data were expressed as mean \pm standard deviation (SD). Qualitative data were expressed as frequency and percentage. Chi-square (x2) test of significance was used in order 
to compare proportions between two qualitative parameters. Risk estimate was calculated using the odds ratio method. Univariate analyses were made to detect the predictors of the presence of biofilm. Multivariate binary logistic regression analysis was used to predict the outcome of categorical variable based on one or more predictor variables. The confidence level was set to $95 \%$ and the margin of error accepted was set to $5 \%$. So, the p-value was considered significant if $<0.05$.

\section{Results}

Both the study group (cases) and the control group are comparable with regard the age and gender. The mean age was $35.8 \pm 7.8$ and $36.3 \pm 7.1$ in the study group and the control group, respectively $(\mathrm{p}$-value $=0.392)$. The male to female ration in both groups was 1:1.

The presence of symptoms was comparable between groups except for the facial pain which was significantly $(\mathrm{p}$-value $=0.025)$ more prevalent in the study group $4(40 \%)$ than in the control group $0(0 \%)$. Other symptoms like nasal obstruction, headache, post-nasal drip and decreased sense of smell were comparable between groups, as shown in Table 1 .

Comparing cases with the control group, there was statistically significant difference ( $\mathrm{p}$-value $=0.001$ ) between them as regard fungal biofilms with prevalence reaching 7 (70\%) in cases while $0(0 \%)$ in controls. By applying risk analysis for fungal biofilm using the odds ratio, there is statistically significant higher risk of positive fungal biofilms in cases of fungal sinusitis, the odds of positive fungal biofilms is far higher in the study group 2.33 than in the control group 0 , (odds ratio is unidentifiable) as shown in Table 2.

Further statistical analyses were made to detect the predictors for the presence of positive fungal biofilms. When using univariate analysis to study the different factors that may predict the presence of positive fungal biofilms, only primary versus recurrent fungal CRS and invasive versus non-invasive fungal CRS were statistically significant (p-values $0.037 \& 0.024$, respectively).

Fungal biofilms are present more in the primary fungal CRS 4 (57.1\%) than in the recurrent fungal CRS $3(42.9 \%)(\mathrm{p}=0.037)$. Also, fungal biofilms are present more in the non-invasive fungal CRS 6 (85.7\%) than in the invasive fungal CRS $1(14.3 \%)$ (p-value $=0.024)$. Other factors like gender, nasal obstruction, and decreased sense of smell, headache, post-nasal drip, complications, and type as acute fulminant, chronic granulomatous, fungal ball or allergy were comparable between those with positive biofilm and negative biofilm, as shown in Table 3 .

Multivariable binary logistic regression analysis was used to predict the outcome of categorical variables based on one or more predictor variables. Regression for the relation between the presence of fungal biofilms with recurrence and invasiveness shows that the lower is the invasiveness, the higher is the positive fungal biofilms ( $\mathrm{OR}=0.94,95 \% \mathrm{CI} 0.46-4.05)$. Also, the analysis showed that the lower is the recurrence the lower is the positive fungal biofilms $(\mathrm{OR}=2.25$, $95 \%$ CI 0.15 - 7.93). 
Table 1. Baseline characteristics.

\begin{tabular}{cccc}
\hline & Study group & Control group & \multirow{2}{*}{ p-value } \\
\cline { 2 - 3 } & $(\mathrm{n}=10)$ & $(\mathrm{n}=10)$ & \\
\hline Age, years (mean \pm SD) & $35.8 \pm 7.8$ & $36.3 \pm 7.1$ & 0.392 \\
Male:Female & $5: 5$ & $5: 5$ & 1.00 \\
Nasal obstruction, n (\%) & $9(90)$ & $7(100)$ & 0.305 \\
Facial pain, n (\%) & $4(40)$ & $0(0)$ & $0.025^{*}$ \\
Decreased sense of smell, n (\%) & $7(70)$ & $5(50)$ & 0.361 \\
Headache, n (\%) & $2(20)$ & $1(10)$ & 0.531 \\
Post nasal drip, n (\%) & $1(10)$ & $0(0)$ & 0.305 \\
\hline
\end{tabular}

Table 2. Risk estimate of fungal biofilms.

\begin{tabular}{cccc}
\hline & Study group & Control group & \multirow{2}{*}{ p-value } \\
\cline { 2 - 3 } & $(\mathrm{n}=10)$ & $(\mathrm{n}=10)$ & \\
\hline Fungal biofilms +ve & 7 & 0 & 0.001 \\
Fungal biofilms -ve & 3 & 10 & \\
Odds of +ve biofilms & $7 / 3=2.33$ & $0 / 10=0$ & \\
Odds ratio & $2.33 / 0=$ unidentifiable & & \\
\hline
\end{tabular}

Table 3. Univariate analysis of predictors of fungal biofilm.

\begin{tabular}{|c|c|c|c|}
\hline \multirow{3}{*}{ Parameters } & Negative biofilm & Positive biofilm & \multirow{3}{*}{$\mathrm{p}$-value } \\
\hline & $(\mathrm{n}=3)$ & $(\mathrm{n}=7)$ & \\
\hline & n (\%) & n (\%) & \\
\hline \multicolumn{4}{|l|}{ Gender } \\
\hline Female & $1(33.3)$ & $4(57.1)$ & 0.49 \\
\hline Male & $2(66.7)$ & $3(42.9)$ & \\
\hline Nasal obstruction & $3(100)$ & $6(85.7)$ & 0.49 \\
\hline Facial pain & $1(33.3)$ & $3(42.9)$ & 0.778 \\
\hline Decreased sense of smell & $3(100)$ & $4(57.1)$ & 0.175 \\
\hline Headache & $0(0)$ & $2(28.6)$ & 0.301 \\
\hline Post nasal drip & $0(0)$ & $1(14.3)$ & 0.49 \\
\hline \multicolumn{4}{|l|}{ Primary/ recurrent } \\
\hline Primary & $3(100)$ & $4(57.1)$ & $0.037^{\star}$ \\
\hline Recurrent & $0(0)$ & $3(42.9)$ & \\
\hline Invasive & $2(66.7)$ & $1(14.3)$ & $0.024^{*}$ \\
\hline Non-invasive & $1(1.33)$ & $6(85.7)$ & \\
\hline Acute fulminant & $0(0)$ & $0(0)$ & 1 \\
\hline Chronic granulomatous & $2(66.7)$ & $1(14.3)$ & 0.098 \\
\hline Fungal ball & $0(0)$ & $1(14.3)$ & 0.49 \\
\hline Allergic & $1(33.3)$ & $5(71.4)$ & 0.26 \\
\hline Complications & $1(33.3)$ & $1(14.3)$ & 0.49 \\
\hline
\end{tabular}

$\mathrm{P}$-value $>0.05$ (non significant) ${ }^{*} \mathrm{p}$-value $<0.05$ (significant). 


\section{Discussion}

The aim of this current gender-matched, age-matched case-control study was to detect the presence of fungal biofilm, in patients with fungal sinusitis trying to find its role in recurrence of fungal sinus infection and resistance to medical treatment. To our knowledge, this study is the first to concentrate on detection of the fungal biofilm in different types of fungal sinusitis whether primary or recurrent, also whether invasive or non-invasive. The study included 10 different cases of fungal sinusitis controlled by 10 cases of non-fungal sinusitis.

As regard the method of biofilm detection, we used SEM for detection of biofilm like other studies addressing the same issue [9] [10]. As regard technique of preparation, in our study, we depended on preparation upon dehydration then tissues were further mounted on brass studs with aluminum conducting tape. This agrees with most of the studies using SEM [9] [11]. However, Sanclement and his colleagues demonstrated that possible limitation of SEM is that preparation of the specimen involves dehydration and may cause protein cross-links that give rise to artifacts similar to biofilms. Dehydration reduces the size of biofilms and minimally distorts its architecture; it remains, however, easily recognizable, as demonstrated by comparison with cryofixation [12] [13].

In this study we did the classic technique in preparation of specimen which was discussed before.

The presence of biofilms on the mucosa of patients with CRS offers a possible cause of antimicrobial therapy failure and could change the approach to treatment. Sanderson et al. (2006) add FISH analysis to detect the causative organism. Also, biopsies in Sanderson study were taken from any patient with CRS and he concluded that biofilm can be the cause of antimicrobial failure. Finally, Sanderson found biofilms in control samples in contrast to our study in which control samples were negative for biofilm [14] [15].

Comparing cases with controls, there was statistically significant difference between them as regard Biofilm ( $\mathrm{p}$-value $=0.001)$ with prevalence reaching (70\%) in cases while (0\%) in controls indicating higher risk of positive biofilm in cases of fungal sinusitis.

Our study results agreed in presence of fungal biofilm in CRS patients with Healy et al. (2008) who first reported a coexistence of fungi and bacterial biofilms in their study of 11 CRS patients. Seven of 11 (64\%) patients had fungal elements within bacterial biofilms [15]. Also, Hochstim et al. (2010) reported similar findings, with 3 of the 15 biofilm-positive patients in their study demonstrating detectable fungal elements [16].

In addition, our study results agreed with Foreman et al. (2009) who have demonstrated robust fungal biofilms in 11 of 50 (22\%) of CRS patients, with a significant association between fungal biofilms and S. aureus biofilms ( 7 of 11, 64\%) [4]. Again, fungal biofilms were observed in 21.9\% (14/64) mucosal samples from patients with sinus fugal ball and none in controls $(\mathrm{P}=0.019)$ in the study made by Wang et al. (2014) but our results were different from them as we 
did not detect bacterial biofilms [17].

As regard selection of patients, in our study we classify study group into primary and recurrent group according to that they underwent ESS before or not. This disagrees with many studies of biofilm in cases of CRS in which any patient undergoing sinus surgery was included regardless of his previous surgical history.

The studies have documented biofilm formation in samples taken from patients during functional endoscopic sinus surgery (FESS) and analyzed after that by EM. They obtained intra-operative samples from the ethmoid bullae from five patients undergoing FESS, all of which demonstrated morphologic criteria of biofilms on EM [9] [18].

In studies made by Prince et al. (2008) the largest study to date and Sanclement et al. (2005), no conditions were put to select patients unlike our study in which there were two groups (primary and recurrent). In these studies, any patient underwent sinus surgery was included in study [12] [19].

The results of the current study showed that there is a statistically significant difference between primary and recurrent fungal sinusitis as regard positive biofilm indicating more affection in recurrent cases. Also, positive biofilm was more in non-invasive CRS than in the invasive CRS.

In agreement to the results of the current study, Loussert et al. (2010) proposed that biofilm formation is claimed to be involved in the pathogenesis of localized as well as invasive diseases caused by Aspergillus fumigatus [6]. On the other hand, detection of biofilm in non-invasive cases disagree with the study done by Singh et al. (2011) which highlights the potential for biofilm formation by at least three medically important species of zygomycetes responsible for mucormycosis [19].

In conclusion, this study showed the presence of fungal biofilms in different cases of fungal sinusitis whether primary or recurrent, also whether invasive or non-invasive. Before, we can recommend that management of any case proven to be fungal sinusitis should include treatment of fungal biofilms, further study with large sample size is recommended to provide strong evidence, as this current one has small number due to the rarity of the condition

\section{Conclusion}

The best procedure for treating OSA varies from patient to patient and there is no universal cure-all. Careful patient selection and pre-operative evaluation are mandatory.

\section{Conflicts of Interest}

The authors declare no conflicts of interest regarding the publication of this paper.

\section{References}

[1] Fastenberg, J.H., Hsueh, W.D., Mustafa, A., Akbar, N.A. and Abuzeid, W.M. (2016) Biofilms in Chronic Rhinosinusitis: Pathophysiology and Therapeutic Strategies. 
World Journal of Otorhinolaryngology-Head and Neck Surgery, 2, 219-229. https://doi.org/10.1016/j.wjorl.2016.03.002

[2] Wolcott, R., Costerton, J.W., Raoult, D. and Cutler, S.J. (2013) The Polymicrobial Nature of Biofilm Infection. Clinical Microbiology and Infection, 19, 107-112.

[3] Ramage, G. and Williams, C. (2013) The Clinical Importance of Fungal Biofilms. In: Advances in Applied Microbiology, Vol. 84, Elsevier, Amsterdam, 27-83. https://doi.org/10.1016/B978-0-12-407673-0.00002-3

[4] Foreman, A., Psaltis, A.J., Tan, L.W. and Wormald, P.J. (2009) Characterization of Bacterial and Fungal Biofilms in Chronic Rhinosinusitis. American Journal of Rhinology \& Allergy, 23, 556-561.

[5] Seidler, M.J., Salvenmoser, S. and Muller, F.M. (2008) Asperigillus fumigatus Forms biofilms with Reduced Antifungal Drug Susceptibility on Bronhial Epithelial Cells. Antimicrobial Agents and Chemotherapy, 52, 4130-4136. https://doi.org/10.1128/AAC.00234-08

[6] Loussert, C., Schmitt, C., Prevost, M.C., Balloy, V., Fadel, E., Philippe, B., Kauffmann-Lacroix, C., Latgé, J.P. and Beauvais, A. (2010) In Vivo Biofilm Composition of Aspergillus fumigatus. Cellular Microbiology, 12, 405-410. https://doi.org/10.1111/j.1462-5822.2009.01409.x

[7] Collins, M.M., Nair, S.B. and Wormald, P.J. (2003) Prevalence of Noninvasive Fungal Sinusitis in South Australia. American Journal of Rhinology \& Allergy, 17, 127-132. https://doi.org/10.1177/194589240301700303

[8] Boase, S., Jervis-Bardy, J., Cleland, E., Pant, H., Tan, L. and Wormald, P.J. (2013) Bacterial-Induced Epithelial Damage Promotes Fungal Biofilm Formation in a Sheep Model of Sinusitis. International Forum of Allergy \& Rhinology, 3, 341-348. https://doi.org/10.1002/alr.21138

[9] Sanclement, J.A., Webster, P., Thomas, J. and Ramadan, H.H. (2005) Bacterial Biofilms in Surgical Specimens of Patients with Chronic Rhinosinusitis. The Laryngoscope, 115, 578-582. https://doi.org/10.1097/01.mlg.0000161346.30752.18

[10] Bezerra, T.F., Piccirillo, J.F., Fornazieri, M.A., Pilan, R.R., Abdo, T.R., Pinna, F.D., Padua, F.G. and Voegels, R.L. (2011) Cross-Cultural Adaptation and Validation of SNOT-20 in Portuguese. International Journal of Otolaryngology, 2011, Article ID: 306529. https://doi.org/10.1155/2011/306529

[11] Boda, S.K., Broda, J., Schiefer, F., Weber-Heynemann, J., Hoss, M., Simon, U., Basu, B. and Jahnen-Dechent, W. (2015) Cytotoxicity of Ultrasmall Gold Nanoparticles on Planktonic and Biofilm Encapsulated Gram-Positive Staphylococci. Small, 11, 3183-3193. https://doi.org/10.1002/smll.201403014

[12] Psaltis, A.J., Weitzel, E.K., Ha, K.R. and Wormald, P.J. (2008) The Effect of Bacterial Biofilms on Post-Sinus Surgical Outcomes. American Journal of Rhinology \& Allergy, 22, 1-6. https://doi.org/10.2500/ajr.2008.22.3119

[13] Sanderson, A.R., Leid, J.G. and Hunsaker, D. (2006) Bacterial Biofilms on the Sinus Mucosa of Human Subjects with Chronic Rhinosinusitis. The Laryngoscope, 116, 1121-1126. https://doi.org/10.1097/01.mlg.0000221954.05467.54

[14] Healy, D.Y., Leid, J.G., Sanderson, A.R. and Hunsaker, D.H. (2008) Biofilms with Fungi in Chronic Rhinosinusitis. Otolaryngology-Head and Neck Surgery, 138, 641-647. https://doi.org/10.1016/j.otohns.2008.02.002

[15] Hochstim, C.J., Masood, R. and Rice, D.H. (2010) Biofilm and Persistent Inflammation in Endoscopic Sinus Surgery. Otolaryngology-Head and Neck Surgery, 143, 697-698. https://doi.org/10.1016/j.otohns.2010.07.017 
[16] Wang, X., Du, J. and Zhao, C. (2014) Bacterial Biofilms Are Associated with Inflammatory Cells Infiltration and the Innate Immunity in Chronic Rhinosinusitis with or without Nasal Polyps. Inflammation, 37, 871-879.

https://doi.org/10.1007/s10753-013-9807-8

[17] Harvey, R. and Lund, V. (2007) Biofilms and Chronic Rhinosinusitis: Systematic Review of Evidence, Current Concepts and Directions for Research. Rhinology, 45, 3-13.

[18] Prince, A.A., Steiger, J.D., Khalid, A.N., Dogrhamji, L., Reger, C., Eau Claire, S., et al. (2008) Prevalence of Biofilm-Forming Bacteria in Chronic Rhinosinusitis. American Journal of Rhinology \& Allergy, 22, 239-245. https://doi.org/10.2500/ajr.2008.22.3180

[19] Singh, R., Shivaprakash, M. and Charkrabartie, A. (2011) Biofilm Formation by Zygomycetes: Quantification, Structure and Matrix Composition. Microbiology, 157, 2611-2618. https://doi.org/10.1099/mic.0.048504-0 